\title{
New Chicane Magnet Design for Insertion Device Straights at the Advanced Light Source
}

\author{
S. Marks, R. Schlueter, D. Anderson, W. Gath, J. Y. Jung, D. Robin, C. Steier, T. Stevens
}

\begin{abstract}
${ }^{1}$ Abstract-- A chicane magnet incorporating counter-rotating permanent magnet pairs together with trim coils has been designed for use in the Advanced Light Source (ALS) straights in conjunction with two insertion devices. In particular, this design is being developed for use in the existing beam line (BL) 4 elliptically polarizing undulator (EPU) straight and in the BL11 EPU straight, currently under design and construction. The purpose of the chicane is to provide a fixed angular separation between two successive EPU photon fans, and to correct steering perturbations resulting from EPU polarization state changes. Polarization changes occur on the time scale of one second; associated steering corrections must be accomplished in less than a second. Hysteresis associated with conventional iron core electromagnets prevents fast steering correction to the required precision. This consideration motivated the iron-free design presented here.
\end{abstract}

\section{INTRODUCTION}

$\mathrm{T}_{\mathrm{B}}^{\mathrm{H}}$ HE Advanced Light Source (ALS) at the Lawrence Berkeley National Laboratory (LBNL) includes ten straight sections designated for insertion devices. A scheme for utilizing two insertion devices in a single straight section uses chicane magnets to produce a sequence of bends in the electron trajectory in order to produce an angular separation in the photon fans from the two insertion devices. This is schematically illustrated in Fig. 1.

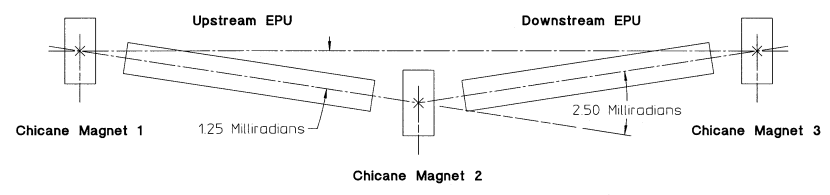

Fig. 1. EPU straight section with chicane magnets bending the electron beam by $1.25 \mathrm{mrad}$ and $2.5 \mathrm{mrad}$.

A facility has been designed to incorporate two elliptically polarizing undulators (EPU's) for use in the magnetic microscopy and spectroscopy experiments at Beam Line (BL)

Manuscript received September 24, 2001. This work was supported by the Director, Office of Energy Research, Office of Basic Energy Sciences, Materials Sciences Division, of the U. S. Department of Energy, under Contract No. DE-AC03-76F00098.

Authors are with Larwence Berkeley National Laboratory, 1 Cyclotron Rd., Berkeley, California, USA.
4 at the ALS [1], [2]. One of the two EPUs is installed, along with three conventional electromagnet chicanes. The chicane magnets must provide the fixed angular bends of approximately 1.25 and $2.5 \mathrm{mrad}$. The field strength must be adjustable to accommodate an electron beam energy range of $1.2 \mathrm{GeV}$ to $1.9 \mathrm{GeV}$, and to provide steering correction to compensate for orbit perturbations produced by integral field errors in the EPU. In particular, the EPU integral errors change with different peak field and polarization states. Corrections must be made in less than a second. Experience with the conventional electromagnet chicanes in the BL4 installation has demonstrated that hysteresis associated with the iron core results in inadequate precision for steering corrections. This paper will describe a chicane magnet designed to avoid hysteresis. It utilizes permanent magnet (PM) rotors for relatively slow gross field adjustments for electron energy change and trim coils for fast steering corrections.

The chicane magnet design described in this paper will be used to replace the center chicane, chicane magnet 2 in Fig. 1, in BL4. The design will also be used in future installations, such as the Molecular and Environmental (MES) beam line currently being designed for installation at BL11 in the ALS. The beam steering correction function at chicane magnets 1 and 3 is being accomplished with arc steering magnets outside the insertion device straight. Therefore, these magnets will not be replaced. For the MES project and future installations, chicanes 1 and 3 will not be required; the fixed bend as well as steering correction will be accomplished with the use of the arc steering magnets.

\section{REQUIREMENTS}

The integrated dipole field required to produce a $2.5 \mathrm{mrad}$ bend is proportional to the electron energy. The primary operating energies for the ALS storage ring are $1.5 \mathrm{GeV}$ and $1.9 \mathrm{GeV}$. The ring is also operated as low as $1.2 \mathrm{GeV}$. The chicane must be able to operate anywhere in this range. The magnet must also have a zero field setting in order to send the electron beam through the insertion device straight without an angular bump. Electrons are injected into the storage ring at $1.5 \mathrm{GeV}$, with $1.9 \mathrm{GeV}$ as the primary operating point. Injection typically occurs every four to eight hours. Energy is ramped from $1.5 \mathrm{Gev}$ to $1.9 \mathrm{GeV}$ in several minutes. The chicane field must ramp through its corresponding range on the same time scale. Table I shows the required integrated 
field values corresponding to a $2.5 \mathrm{mrad}$ bend, for the range of electron energies.

TABLE I

Field INTEGRAL VALUES CORRESPONDING TO 2.5 MRAD BEND

\begin{tabular}{cc}
\hline Electron Energy & $\boldsymbol{\int} \boldsymbol{B} \cdot \boldsymbol{d} \boldsymbol{z}$ for $\mathbf{2 . 5}$ mrad \\
\hline $1.2 \mathrm{GeV}$ & $0.01001 \mathrm{~T}-\mathrm{m}$ \\
$1.5 \mathrm{GeV}$ & $0.01251 \mathrm{~T}-\mathrm{m}$ \\
$1.9 \mathrm{GeV}$ & $0.01584 \mathrm{~T}-\mathrm{m}$ \\
\hline
\end{tabular}

Based upon experience with the first EPU, corrections of $\pm 2.0 \times 10^{-4} \mathrm{~T}-\mathrm{m}$ for vertical steering, and of $\pm 7 \times 10^{-5} \mathrm{~T}-\mathrm{m}$ for horizontal steering correction are required to compensate for changing states of a single EPU. A vertical correction range of $\pm 6.0 \times 10^{-4} \mathrm{~T}-\mathrm{m}$ and a horizontal correction range of $\pm 3.0 \times 10^{-4} \mathrm{~T}$-m will be adequate for two devices in the same straight.

Higher order multipoles meeting the following requirement are considered to be acceptable

$$
\left|\frac{c_{n}}{c_{1}}\right|_{n>1}<1 \times 10^{-3} \text { at } r_{0}=1 \mathrm{~cm} \text {. }
$$

\section{MAGNET DeSIGN}

\section{A. Concept}

Fig 2 schematically illustrates the design concept for the new chicane magnet. Six permanent magnet rotors are shown with magnetic orientations indicated by arrows. Vertical and horizontal coils are indicated with labels VS and HS respectively. Coil returns are schematically illustrated as solid lines for horizontal and dashed lines for vertical steering coils.

Fig. 2. Schematic illustration of chicane magnet design concept.
The PM configuration is based upon a concept for a harmonic corrector ring consisting of PM rotor pairs with centers evenly spaced in azimuth on a radius about the beam axis [3]. In the general case, $M$ rotor pairs can vary orientation and strength to produce a desired mix of multipoles up to the $N t h$ order, where $N<M$. The design being considered here represents a special case, where the intent is to produce a vertical dipole field only. The use of six rotor pairs exceeds the minimum required for this application. However, this configuration is used to produce suitable field strength with very small higher order multipoles. Each rotor in Fig. 2 represents a co-axial pair. Fig. 3 shows the actual mechanical configuration of rotor pairs. The effective strength of each pair is altered by counter-rotation. For example, a vertical magnetic orientation for the uppermost rotor, as is indicated in Fig. 2, is correct for producing the desired vertical dipole. The effective orientation of this pair can be maintained while changing its integrated strength if the two are counter-rotated by angles $\pm \varphi$. For the general case, the effective strength and orientation of each rotor pair can be altered to produce a desired mix of field harmonics [3]. For the restricted dipole only case being considered here, all rotor pairs maintain a fixed effective orientation, and overall strength is altered by equal counter-rotation of all rotor pairs. The details of the PM design will be discussed in Section $B$ below.

\section{B. PM Design}

The general equation for the integrated field, represented as a complex function, is given by [3]

$$
\begin{aligned}
& I^{*}(z)=\sum_{n} c_{n}\left(\frac{z}{r_{0}}\right)^{n-1}=B_{r} L \sum_{n} \frac{n r_{c}^{2}}{2 R^{n+1}} \sum_{m=0}^{M-1} e^{-i(n+1) \beta_{m}} e^{i \varphi_{m}} ; . \\
& z=x+i y,
\end{aligned}
$$

where definitions of the terms, and their values for this design (indicated in parentheses), are as follows:

$$
\begin{aligned}
& B_{r} \equiv \text { PM remnant field }(1.28 \mathrm{~T}), \\
& L / 2 \equiv \text { effective length of single rotors }(3.5 \mathrm{~cm}), \\
& n \equiv \text { multipole number, } \\
& r_{c} \equiv \text { radius of rotors }(4.064 \mathrm{~cm}), \\
& R \equiv \text { assembly axis to rotor center radius }(7.5 \mathrm{~cm}), \\
& m \equiv \text { rotor number, } \\
& M \equiv \text { total number of rotors }(6), \\
& \beta_{m} \equiv \text { azimuth angle for individual rotors } \\
& ((2 m+1) \pi / M), \\
& \varphi_{m} \equiv \text { orientation angle for individual rotors }\left(2 \beta_{m}-\pi / 2\right)
\end{aligned}
$$

Using this equation with the above values, the maximum integrated dipole, $n=1$, is $0.02 \mathrm{~T}-\mathrm{m}$.

In the ideal case all rotors have equal strength and angles $\beta_{m}$ and $\varphi_{m}$ are set as indicated. The first allowed higher order multipole will be $n=7$. Its normalized strength at a radius of $r_{0}=1 \mathrm{~cm}$ will be

$$
\frac{b_{7}}{b_{1}}=7\left(\frac{1}{7.5}\right)^{6}=4 \times 10^{-5} \text {. }
$$


Now let us consider perturbations, or deviations from the ideal case. First, consider a rotor strength perturbation. This may be caused by variations in the parameters $B_{r}, L, \varphi_{m}$, or combinations thereof. For strength perturbation $\Delta\left(B_{r} L\right)$, the following error multipoles are generated

$$
\left|\frac{c_{n}}{c_{1}}\right|=\frac{n \Delta\left(B_{r} L\right)}{B_{r} L}\left(\frac{r_{0}}{R}\right)^{n-1} .
$$

Using the parameter values given above, for $n=2$ (the worst case), the multipole requirement is met for

$$
\left|\frac{\Delta\left(B_{r} L\right)}{B_{r} L}\right|<0.02 \text {. }
$$

For a perturbation in the radial position of a rotor, $\Delta R$, the following multipole errors are generated

$$
\left|\frac{c_{n}}{c_{1}}\right|=\frac{-n(n+1) r_{0}^{n-1}}{6 R^{n}} \Delta R .
$$

Multipole requirements for this case are met for

$$
\left|\frac{\Delta R}{R}\right|<7.5 \times 10^{-3} \text {. }
$$

An error in rotor azimuth $\Delta \beta$ generates multipoles

$$
\left|\frac{c_{n}}{c_{1}}\right|=i n(1-n)\left(\frac{r_{0}}{R}\right)^{n-1} \Delta \beta \text {. }
$$

Multipole requirements are met for

$$
|\Delta \beta|<0.02=1.3^{\circ} \text {. }
$$

The mechanical tolerances associated with set of errors considered above are all relatively easy to achieve.

Errors in relative orientation of rotors will result in a set of error multipoles. The theory developed for the Harmonic Corrector Ring [3] can be used to determine orientation errors from measured multipoles. A rotating search coil be used to measure integral multipoles. These will then be used as the basis for adjusting relative rotor orientations.

\section{Coil Design}

The horizontal and vertical steering coils must meet the field strength and multipole requirements stated in Section II above. Design analysis, based upon two-dimensional complex equations for current elements, was used to calculate required currents and multipoles. The affect of finite coil cross sections and three-dimensional coil return radii will result in multipoles slightly different from those calculated. However, since coil cross sections are small and steering fields are a small components of the overall magnet field, these deviations are negligible.

\section{1) Horizontal Steering Coil}

The two-dimensional model consists of current elements with $+I$ total current at locations $z=z_{0}=x_{0}+i y_{0}$ and $z=-z_{0}^{*}$. Elements with $-I$ total current are at $z=z_{0}^{*}$ and $z=-z_{0}$. The field equation for this current distribution is

$$
\begin{aligned}
B^{*} & \equiv B_{x}+i B_{y} \\
& =\sum_{n}-\frac{u_{0} I}{\pi i}\left[1+(-1)^{n}\right]\left(\frac{1}{y_{0}}\right)^{n+1} \sin ^{n+1} \theta_{0} \cos (n+1) \theta_{0} \\
\theta_{0} & =\tan ^{-1} \frac{y_{0}}{x_{0}}
\end{aligned}
$$

The angle $\theta_{0}=30^{\circ}$ is used since this results in a null of the first allowed multipole, $n=3$. The equation for the dipole term is

$$
B_{1}^{*}=-\frac{\mu_{0} I}{\pi i} \frac{2}{y_{0}} \sin \theta_{0} \cos \theta_{0} .
$$

A total current of $I=100$ A-turns produces the required $3.0 \times 10^{-4} \mathrm{~T}-\mathrm{m}$ integrated dipole. This is based upon the value $y_{0}=1.5 \mathrm{~cm}$, required for vacuum chamber clearance, and a $13 \mathrm{~cm}$ coil length, resulting in $\left|B_{1}^{*}\right|=2.3 \times 10^{-3} \mathrm{~T}$. This is accomplished using a $3 \times 3$ coil cross section with $\# 12$ square solid conductor. The required current is $11 \mathrm{~A}$.

\section{2) Vertical Steering Coil}

The two-dimensional model consists of current elements with $+I$ total current at locations $z=z_{0}=x_{0}+i y_{0}$ and $z=z_{0}^{*}$. Elements with $-I$ total current are at $z=-z_{0}$ and $z=-z_{0}^{*}$. The field equation for this current distribution is

$$
B^{*}=\sum_{n} \frac{u_{0} I}{\pi i}\left[1+(-1)^{n}\right]\left(\frac{1}{y_{0}}\right)^{n+1} \sin ^{n+1} \theta_{0} \sin (n+1) \theta_{0} .
$$

The angle $\theta_{0}=60^{\circ}$ is used since this results in a null of the first allowed multipole, $n=3$. The equation for the dipole term is

$$
B_{1}^{*}=-\frac{\mu_{0} I}{\pi i} \frac{2}{y_{0}} \sin ^{2} \theta_{0} .
$$

A total current of $I=200$ A-turns produces the required $6.0 \times 10^{-4} \mathrm{~T}-\mathrm{m}$ integrated dipole. This is based upon the value $y_{0}=3 \mathrm{~cm}$, and a $15 \mathrm{~cm}$ coil length, resulting in $\left|B_{1}^{*}\right|=4.0 \times 10^{-3} \mathrm{~T}$. A $4 \times 4$ coil cross section of $\# 11$ round solid conductor is used. The required current is $12.5 \mathrm{~A}$. Round wire is used for these coils because of the difficulty of routing returns, as is illustrated in Fig. 3 below.

\section{Mechanical Design}

Fig. 3 shows the internal structure of the rotors and coils. The horizontal steering coils are simple planar windings. The additional complexity of the vertical steering coil winding is dictated by the current path and clearance requirements.

The PM rotors are enclosed in aluminum canisters; rotors are shimmed to provide a snug fit. The rotors are indexed to the canisters via machined grooves in the rotors which are captured by dowel pins.

Fig. 4 shows the full magnet assembly installed around its section of the ALS storage ring. The rotors are driven by sets of chains and sprockets. Two micro-stepping motors, with 
rotary encoders, are used, one for each rotor set, front and back. A gear ratio of $6: 1$ is used between the motor and rotors. The magnet assembly is mounted to a rail table with a drive motor so that it can be retracted from around the vacuum chamber.

The rotors and coils are supported by two outer frame plates and a center support plate. Fig. 3 shows the center plate and one of the outer frame plates. The rotors are supported via bearings. The coils are held in place via clips.

Sprockets are attached to the rotor axes with miniature clamps. This allows the relative orientation of individual rotors to be adjusted. An individual rotor orientation is adjusted by loosening the sprocket clamp while adjusting the rotor with a wrench on the hex head machined on the rotor shaft. Angle veneers are attached to the rotors and the adjacent frame body for precise angular adjustment. After relative orientations have been set, using magnetic measurements, rotor sprockets will be pinned to prevent further movement.
The chain and sprocket arrangement prevents relative rotation of individual rotors within a rotor set. Relative rotation of the front and back rotor sets is used to adjust the dipole strength and rotation. If the two rotor sets are counterrotated by equal angles, the dipole orientation remains fixed while the strength changes. Differential relative rotation will also result in a shift in the dipole orientation. However, as long as rotor angles within the two sets are fixed, rotation will not produce new multipoles.

\section{CONCLUSION}

Final assembly is nearly complete for two chicane magnets. Magnetic measurements and magnet adjustments will follow. The first will replace the existing center chicane magnet for the BL4 facility. The second will be installed for the new BL11 MES facility.

\section{REFERENCES}

[1] A. Young, et al., 'Elliptically polarizing undulator beamlines at the Advanced Light Source', Rev. Sci. Instrum., 67 (1996), 3372.

[2] S. Marks, et al., 'The Advanced Light Source Elliptically Polarizing Undulator', Proceedings 1997 U.S. Particle Accelerator Conference.

[3] R. D. Schlueter, D. Humphries, J. Tanabe, 'Pure Permanent Magnet Harmonics Corrector Ring', Nucl. Instr. Meth. A395 (1997), 153-158.

Fig. 3. Rotor and coil configuration

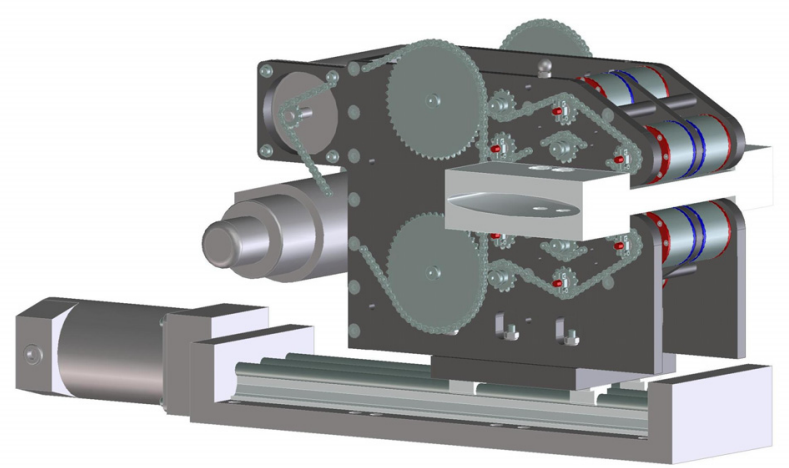

Fig. 4. Full chicane magnet assembly. 